PROCEEDINGS OF THE

AMERICAN MATHEMATICAL SOCIETY

Volume 140, Number 4, April 2012, Pages 1353-1362

S 0002-9939(2011)11172-9

Article electronically published on August 4, 2011

\title{
NON-EVEN LEAST ENERGY SOLUTIONS OF THE EMDEN-FOWLER EQUATION
}

\author{
RYUJI KAJIKIYA
}

(Communicated by Yingfei Yi)

\begin{abstract}
In this paper, we study the Emden-Fowler equation whose coefficient is even in the interval $(-1,1)$ under the Dirichlet boundary condition. We prove that if the density of the coefficient function is thin in the interior of $(-1,1)$ and thick on the boundary, then a least energy solution is not even. Therefore the equation has at least three positive solutions: the first one is even, the second one is a non-even least energy solution $u(t)$ and the third one is the reflection $u(-t)$.
\end{abstract}

\section{IntRODUCTION AND MAIN RESULTS}

We study non-even positive solutions of the Emden-Fowler equation

$$
\begin{aligned}
& u^{\prime \prime}+h(t) u^{p}=0 \quad \text { in }(-1,1), \\
& u>0 \quad \text { in }(-1,1), \\
& u(-1)=u(1)=0,
\end{aligned}
$$

where $p>1, h \in L^{\infty}(-1,1), h(t)$ is even and $h(t) \geq 0, \not \equiv 0$. If $0<p<1$, Brezis and Oswald [2] proved that there exists a unique positive solution. Therefore we study the case $p>1$. Since $h(t)$ is assumed to be even, there exists an even positive solution, which will be proved later on. However, in this paper we expect the existence of a non-even positive solution under a suitable condition on $h(t)$. This solution will be given by a least energy solution. To explain it, we define the Rayleigh quotient

$$
R(u):=\left(\int_{-1}^{1} u^{\prime}(t)^{2} d t\right) /\left(\int_{-1}^{1} h(t)|u|^{p+1} d t\right)^{2 /(p+1)}
$$

and the least energy

$$
L:=\inf \left\{R(u): u \in H_{0}^{1}(-1,1) \backslash\{0\}\right\} .
$$

Here $H_{0}^{1}(-1,1)$ denotes the Sobolev space. Because of the Sobolev imbedding theorem, $R(u)$ has a positive lower bound. Hence $L$ is well defined and positive.

Received by the editors January 4, 2011.

2010 Mathematics Subject Classification. Primary 34B15, 34B18.

Key words and phrases. Emden-Fowler equation, least energy solution, non-even positive solution, variational method.

The author was supported in part by Grant-in-Aid for Scientific Research (C) (No. 20540197), Japan Society for the Promotion of Science.

(C)2011 American Mathematical Society Reverts to public domain 28 years from publication 
We define the Nehari manifold

$$
\mathcal{N}:=\left\{u \in H_{0}^{1}(-1,1) \backslash\{0\}: \int_{-1}^{1}\left(\left|u^{\prime}\right|^{2}-h(t)|u|^{p+1}\right) d t=0\right\} .
$$

Note that for any $u \in H_{0}^{1}(-1,1) \backslash\{0\}$, there is a $\lambda>0$ such that $\lambda u \in \mathcal{N}$. Since $R(\lambda u)=R(u)$ for any $\lambda>0$, it holds that

$$
\begin{aligned}
L & =\inf \left\{R(u): u \in H_{0}^{1}(-1,1) \backslash\{0\}\right\} \\
& =\inf \{R(u): u \in \mathcal{N}\} .
\end{aligned}
$$

We call $u$ a least energy solution if $u \in \mathcal{N}$ and $R(u)=L$. It is easy to verify that a least energy solution satisfies (1.3) and (1.1) with the non-linear term $h(t) u^{p}$ replaced by $h(t)|u|^{p-1} u$. Moreover, it is sign-definite; i.e., it is positive or negative in $(-1,1)$. Indeed, if $u \in \mathcal{N}$ is a least energy solution, then $|u| \in \mathcal{N}$. Hence $|u|$ is a minimizer of $R$ over $\mathcal{N}$, and it is also a solution of (1.1) and (1.3). Then $|u|$ is positive by the strong maximum principle. Accordingly, $u$ is a positive or negative solution. Throughout this paper, a least energy solution means a positive one.

To explain our motivation, we introduce the Hénon equation

$$
-\Delta u=|x|^{\lambda} u^{p}, \quad u>0 \quad \text { in } B, \quad u=0 \quad \text { on } \partial B,
$$

where $B$ denotes the unit ball in $\mathbb{R}^{N}$ and $1<p<\infty$ if $N=1,2$ and $1<p<$ $(N+2) /(N-2)$ if $N \geq 3$. Then Smets, Willem and $\mathrm{Su}[10$ ] have proved that if $\lambda$ is large enough, then a least energy solution of (1.4) is non-radial. Therefore the equation has at least two positive solutions: one is a least energy solution and another is a radial solution. There are many contributions that have studied the Hénon equation $([3,4,5,6,6,9])$.

On the other hand, Moore and Nehari 7$]$ have proved that if $h(t)=0$ in $[-a, a]$, $h(t)=1$ in $[-1,-a) \cup(a, 1]$ and $a(<1)$ is sufficiently close to 1 , then (1.1)-(1.3) has at least three positive solutions: the first one is even, the second one $u(t)$ is non-even and the third one is the reflection $u(-t)$. Tanaka [11, 12] has extended the results above to sign-changing solutions of the $p$-Laplace equation and to radial solutions of a semilinear elliptic equation.

Observing the results above, we have a question: What kind of $h(t)$ yields a non-even least energy solution? Our answer is as follows. If the density of $h(t)$ is thin in the interval $(-a, a)$ with $a$ close to $t=1$ and concentrates on the boundary $t= \pm 1$, then a least energy solution is not even.

To state our results, we put

$$
H:=\left(\int_{0}^{1} h(t)(1-t)^{p+1} d t\right)\left(\int_{0}^{1} h(t) d t\right)^{-1}
$$

and introduce two assumptions below:

(A) There exist $\mu \in[0,1)$ and $a \in(0,1)$ such that

$$
\begin{gathered}
(a(1-a))^{(p+1) / 2} \int_{0}^{a} h(t) d t \leq \mu^{p+1} \int_{a}^{1} h(t)(1-t)^{p+1} d t, \\
\frac{1-a}{(1-\mu)^{2}} \leq 2\left(\frac{H+1}{2}\right)^{2 /(p+1)}-1 .
\end{gathered}
$$


(B) There exist $\nu \in[0,1)$ and $a \in(0,1)$ such that

$$
\begin{gathered}
(2(1-a))^{(p+1) / 2} \int_{0}^{a} h(t)(a-t)^{(p+1) / 2} d t \\
\leq \nu^{(p+1) / 2} \int_{a}^{1} h(t)(1-t)^{p+1} d t, \\
\frac{1-a}{1-\nu} \leq 2\left(\frac{H+1}{2}\right)^{2 /(p+1)}-1 .
\end{gathered}
$$

Theorem 1.1. Let $1<p<\infty$. Assume that $h \in L^{\infty}(-1,1), h \geq 0, \not \equiv 0, h(t)$ is even and satisfies either Assumption (A) or (B). Then a least energy solution of (1.1) -(1.3) is not even. Therefore (1.1)-(1.3) has at least three positive solutions: the first one is even, the second one is a non-even least energy solution $u(t)$ and the third one is the reflection $u(-t)$.

In the theorem above, we assume that $h \in L^{\infty}(-1,1)$. Then a solution of (1.1) belongs to $W^{2, \infty}(-1,1)$ and satisfies (1.1) almost everywhere. If $h$ is in $C[-1,1]$, then a solution belongs to $C^{2}[-1,1]$ and becomes a classical solution. By Theorem 1.1, we have the next corollary.

Corollary 1.2. Impose the assumptions of Theorem 1.1 with (1.5) (1.9) replaced by

$$
\begin{gathered}
2^{(p+1) / 2} \int_{0}^{a} h(t)(1-t)^{p+1} d t \leq \nu^{(p+1) / 2} \int_{a}^{1} h(t)(1-t)^{p+1} d t \\
\frac{1-a}{1-\nu} \leq 2^{(p-1) /(p+1)}-1
\end{gathered}
$$

with some $\nu \in[0,1)$ and $a \in(0,1)$. Then the same conclusion as in Theorem 1.1 remains valid.

The corollary above ensures our assertion that if $a$ is close to 1 and if the density of $h(t)$ is thin in $(-a, a)$ and thick in $(-1,-a) \cup(a, 1)$, then the least energy solution is not even. Our theorem extends and generalizes known results. Indeed, using Corollary 1.2 we get the same result as Moore and Nehari's theorem.

Example 1.3. Let $h(t) \in L^{\infty}(-1,1)$ satisfy that $h(t)$ is even, non-negative and that $h(t) \leq \varepsilon$ for $|t| \leq 1-2 \delta$ and $h(t)=1$ for $1-\delta \leq|t| \leq 1$. Here $0 \leq \varepsilon \leq \delta^{p+2+c}$ with a constant $c>0$. If $\delta>0$ is small enough, then the same conclusion as in Theorem 1.1 holds.

The example above and the next one will be verified in Section 4 .

Example 1.4. Let $g \in C[-1,1]$ satisfy the fact that $g(t)$ is even and $0 \leq g(t)<$ $g( \pm 1)$ for $|t|<1$. Put $h(t)=g(t)^{\lambda}$. If $\lambda>0$ is large enough, then the same conclusion as in Theorem 1.1 holds.

The example above is a new result which covers a wide class of $h(t)$. Indeed, it is applicable to $h(t)=e^{\lambda|t|}, h(t)=|\sin (\pi t / 2)|^{\lambda}, h(t)=(|t| /(1+|t|))^{\lambda}$, etc. Moreover, putting $g(t)=|t|$, we obtain the result on the Hénon equation in one dimension as below.

Example 1.5. Let $h(t)=|t|^{\lambda}$. If $\lambda>0$ is large enough, then the same conclusion as in Theorem 1.1 holds. 
This paper is organized as follows. In Section 2, we study the property of even positive solutions, which will be used in Section 3. In Section 3, we prove Theorem 1.1 and Corollary 1.2. In Section 4, we verify Examples 1.3 and 1.4.

\section{Even POSITIVE SOLUTION}

In this section, we study the property of even positive solutions, which will be needed in the next section. For $-1 \leq a<b \leq 1$, we write

$$
R(v ;(a, b)):=\left(\int_{a}^{b} v^{\prime}(t)^{2} d t\right)\left(\int_{a}^{b} h|v|^{p+1} d t\right)^{-2 /(p+1)} .
$$

Then $R(v ;(-1,1))$ denotes $R(v)$. If $v(t)$ is even, then we have the relation

$$
R(v)=R(v ;(-1,1))=2^{(p-1) /(p+1)} R(v ;(0,1)) .
$$

Lemma 2.1. There exists an even positive solution.

Proof. This is a consequence of a mountain pass lemma. Indeed, we put

$$
\begin{aligned}
I(u) & :=\int_{-1}^{1}\left(\frac{1}{2} u^{\prime}(t)^{2}-\frac{1}{p+1} h(t)|u|^{p} u\right) d t, \\
H_{0, e}^{1}(-1,1) & :=\left\{u \in H_{0}^{1}(-1,1): u(-t)=u(t)\right\} .
\end{aligned}
$$

$H_{0, e}^{1}(-1,1)$ is a closed linear subspace of $H_{0}^{1}(-1,1)$ consisting of even functions. Moreover, $I$ is a $C^{1}$-functional on $H_{0}^{1}(-1,1)$ which satisfies the Palais-Smale condition. We restrict $I$ on $H_{0, e}^{1}(-1,1)$ and use a mountain pass lemma (see [1] or [13]). Then we get a non-trivial critical point of $I$ in $H_{0, e}^{1}(-1,1)$, which is a solution to

$$
-u^{\prime \prime}=h|u|^{p} \geq 0 \quad \text { in }(-1,1), \quad u(-1)=u(1)=0 .
$$

By the strong maximum principle, $u$ is positive. Thus $u$ is an even positive solution.

Lemma 2.2. Let $\phi$ be an even positive solution of (1.1)-(1.3). Then we have:

(i) $\phi^{\prime}(t), \phi^{\prime \prime}(t) \leq 0$ in $(0,1), \phi^{\prime}(0)=0$ and $\phi(t)$ attains its maximum at $t=0$,

(ii) $\int_{0}^{1}\left|\phi^{\prime}\right|^{2} d t=\int_{0}^{1} h \phi^{p+1} d t$,

(iii) $R(\phi ;(0,1))=\left(\int_{0}^{1}\left|\phi^{\prime}\right|^{2} d t\right)^{(p-1) /(p+1)}=\left(\int_{0}^{1} h \phi^{p+1} d t\right)^{(p-1) /(p+1)}$.

Proof. For a positive solution $\phi$, it holds that $-\phi^{\prime \prime}=h \phi^{p} \geq 0$; i.e., $\phi$ is concave. If $\phi$ is even, then $\phi^{\prime}(0)=0$. Thus (i) follows. Multiplying (1.1) by $\phi$, integrating it over $[0,1]$ and using $\phi^{\prime}(0)=\phi(1)=0$, we have (ii). The assertion (ii) implies (iii) directly.

\section{Proof of the Main Results}

In this section, we prove the main theorems. Let $H_{0, e}^{1}(-1,1)$ be defined by (2.2). We define the even least energy

$$
L_{e}:=\inf \left\{R(u): u \in H_{0, e}^{1}(-1,1) \backslash\{0\}\right\}=\inf \left\{R(u): u \in \mathcal{N}_{e}\right\},
$$

where

$$
\mathcal{N}_{e}:=\mathcal{N} \cap H_{0, e}^{1}(-1,1)
$$


If $u \in \mathcal{N}_{e}$ and $R(u)=L_{e}$, then we call $u$ an even least energy solution. Such a solution exists. We give an upper estimate of the even least energy $L_{e}$.

Lemma 3.1. For any $a \in(0,1)$, it holds that

$$
L_{e} \leq 2^{(p-1) /(p+1)}(1-a)\left(\int_{a}^{1} h(t)(1-t)^{p+1} d t\right)^{-2 /(p+1)} .
$$

Proof. We define a test function

$$
v(t):= \begin{cases}1 & \text { if }|t| \leq a \\ (1-|t|) /(1-a) & \text { if } a \leq|t| \leq 1 .\end{cases}
$$

Then it belongs to $H_{0, e}^{1}(-1,1)$. Since $L_{e} \leq R(v)$ by definition, it is enough to estimate $R(v)$ from above. From the definition of $v(t)$, we have

$$
\begin{gathered}
\int_{0}^{1}\left|v^{\prime}\right|^{2} d t=\frac{1}{1-a} \\
\int_{0}^{1} h|v|^{p+1} d t \geq(1-a)^{-(p+1)} \int_{a}^{1} h(1-t)^{p+1} d t .
\end{gathered}
$$

These inequalities with (2.1) imply

$$
\begin{aligned}
R(v ;(-1,1)) & =2^{(p-1) /(p+1)} R(v ;(0,1)) \\
& \leq 2^{(p-1) /(p+1)}(1-a)\left(\int_{a}^{1} h(t)(1-t)^{p+1} d t\right)^{-2 /(p+1)} .
\end{aligned}
$$

This completes the proof.

Lemma 3.2. Let $\phi$ be an even positive least energy solution. Assume that either (A) or (B) holds. Then we have

$$
\begin{aligned}
\phi(0) & \leq \frac{1}{1-\mu} \phi(a) \quad \text { in Case }(\mathrm{A}), \\
\phi(0)^{2} & \leq \frac{1}{1-\nu} \phi(a)^{2} \quad \text { in Case (B). }
\end{aligned}
$$

Proof. Recall that $\phi^{\prime}(t) \leq 0$ in $(0,1)$ by Lemma 2.2 (i). Multiplying (1.1) by $\phi$ and integrating it over $[0, t]$, we get

$$
\left|\phi^{\prime}(t)\right| \phi(t)+\int_{0}^{t} \phi^{\prime}(s)^{2} d s=\int_{0}^{t} h \phi(s)^{p+1} d s \quad \text { for } 0 \leq t \leq 1 .
$$

First, we assume (A). Putting $t=a$ in (3.4), we have

$$
\int_{0}^{a} \phi^{\prime}(t)^{2} d t \leq \int_{0}^{a} h \phi(t)^{p+1} d t
$$


This inequality with the Schwartz inequality gives us

$$
\begin{aligned}
0 \leq \phi(0)-\phi(a) & \leq\left|\int_{0}^{a} \phi^{\prime}(t) d t\right| \\
& \leq \sqrt{a}\left(\int_{0}^{a} \phi^{\prime}(s)^{2} d s\right)^{1 / 2} \\
& \leq \sqrt{a}\left(\int_{0}^{a} h \phi(s)^{p+1} d s\right)^{1 / 2} \\
& \leq \sqrt{a} \phi(0)\left(\int_{0}^{a} h \phi(s)^{p-1} d s\right)^{1 / 2} .
\end{aligned}
$$

Using the Hölder inequality, we estimate the last integral as

$$
\int_{0}^{a} h \phi(s)^{p-1} d s \leq\left(\int_{0}^{a} h d t\right)^{2 /(p+1)}\left(\int_{0}^{a} h \phi^{p+1} d t\right)^{(p-1) /(p+1)} .
$$

We use Lemma 2.2 (iii) with (2.1) and apply Lemma 3.1 to get

$$
\begin{aligned}
\left(\int_{0}^{a}\right. & \left.h \phi(s)^{p+1} d s\right)^{(p-1) /(p+1)} \\
& \leq R(\phi ;(0,1)) \\
& =2^{-(p-1) /(p+1)} R(\phi ;(-1,1)) \\
& =2^{-(p-1) /(p+1)} L_{e} \\
& \leq(1-a)\left(\int_{a}^{1} h(t)(1-t)^{p+1} d t\right)^{-2 /(p+1)}
\end{aligned}
$$

Combining all the inequalities above, we obtain

$$
\begin{aligned}
\phi(0)-\phi(a) \leq \sqrt{a(1-a)} \phi(0)\left(\int_{0}^{a} h d t\right)^{1 /(p+1)} \\
\times\left(\int_{a}^{1} h(t)(1-t)^{p+1} d t\right)^{-1 /(p+1)} .
\end{aligned}
$$

This inequality with (1.6) ensures (3.2).

We deal with Case (B). By (3.4), we have

$$
\left|\frac{d}{d t} \phi(t)^{2}\right| \leq 2 \int_{0}^{t} h \phi^{p+1} d s .
$$

Integrating both sides over $[0, a]$, we get

$$
\begin{aligned}
0 \leq \phi(0)^{2}-\phi(a)^{2} & \leq 2 \int_{0}^{a}\left(\int_{0}^{t} h \phi(s)^{p+1} d s\right) d t \\
& \leq 2 \phi(0)^{2} \int_{0}^{a}(a-s) h(s) \phi(s)^{p-1} d s .
\end{aligned}
$$


Using the Hölder inequality, we estimate the last integral as

$$
\begin{aligned}
\int_{0}^{a}(a-s) h(s) \phi(s)^{p-1} d s \\
\leq\left(\int_{0}^{a} h(s) \phi(s)^{p+1} d s\right)^{(p-1) /(p+1)} \\
\quad \times\left(\int_{0}^{a} h(s)(a-s)^{(p+1) / 2} d s\right)^{2 /(p+1)} .
\end{aligned}
$$

Combining (3.5)-(3.7), we obtain

$$
\begin{gathered}
\phi(0)^{2}-\phi(a)^{2} \leq 2 \phi(0)^{2}(1-a)\left(\int_{a}^{1} h(t)(1-t)^{p+1} d t\right)^{-2 /(p+1)} \\
\times\left(\int_{0}^{a} h(s)(a-s)^{(p+1) / 2} d s\right)^{2 /(p+1)} .
\end{gathered}
$$

This inequality with (1.8) yields (3.3). The proof is complete.

Lemma 3.3. Under the assumption of Lemma 3.2, we have

$$
\phi(0)^{2} \leq \theta \int_{a}^{1} \phi^{\prime}(t)^{2} d t
$$

where

$$
\theta:= \begin{cases}(1-a) /(1-\mu)^{2} & \text { in Case (A), } \\ (1-a) /(1-\nu) & \text { in Case (B). }\end{cases}
$$

Proof. We use $\phi(1)=0$ and the Schwartz inequality to get

$$
|\phi(a)|=\left|\int_{a}^{1} \phi^{\prime}(t) d t\right| \leq \sqrt{1-a}\left(\int_{a}^{1} \phi^{\prime}(t)^{2} d t\right)^{1 / 2} .
$$

This inequality with Lemma 3.2 leads to the conclusion.

We are now in a position to give a proof of Theorem 1.1. Our method of the proof is to prove $L<L_{e}$. If this inequality can be proved, then a solution corresponding to $L$ cannot be even, that is, a least energy solution is not even.

Proof of Theorem 1.1. Let $\phi$ be an even positive least energy solution. We define a test function

$$
v(t):= \begin{cases}\phi(t) & \text { if } t \in[-1,0], \\ M(1-t) & \text { if } t \in[0,1],\end{cases}
$$

where $M:=\phi(0)$ is a maximum of $\phi(t)$. Then $v$ belongs to $H_{0}^{1}(-1,1)$. We shall estimate the Rayleigh quotient,

$$
R(v)=\left(\int_{-1}^{1} v^{\prime}(t)^{2} d t\right)\left(\int_{-1}^{1} h v^{p+1} d t\right)^{-2 /(p+1)} .
$$


Since $\phi$ is an even positive least energy solution, Lemma 3.3 gives us

$$
\begin{aligned}
\int_{-1}^{1} v^{\prime}(t)^{2} d t & =\int_{-1}^{0} \phi^{\prime}(t)^{2} d t+M^{2} \\
& \leq \int_{0}^{1} \phi^{\prime}(t)^{2} d t+\theta \int_{a}^{1} \phi^{\prime}(t)^{2} d t \\
& \leq(1+\theta) \int_{0}^{1} \phi^{\prime}(t)^{2} d t
\end{aligned}
$$

From the definition of $v(t)$, it follows that

$$
\int_{-1}^{1} h v^{p+1} d t=\int_{-1}^{0} h \phi^{p+1} d t+M^{p+1} \int_{0}^{1} h(1-t)^{p+1} d t .
$$

Since $M=\phi(0)$ is a maximum of $\phi(t)$, by (1.5) we estimate the last term as

$$
M^{p+1} \int_{0}^{1} h(1-t)^{p+1} d t=M^{p+1} H \int_{0}^{1} h d t \geq H \int_{0}^{1} h \phi^{p+1} d t .
$$

Then (3.10) is reduced to

$$
\int_{-1}^{1} h v^{p+1} d t \geq(H+1) \int_{0}^{1} h \phi^{p+1} d t .
$$

In both cases (1.7) and (1.9), we have

$$
2^{-(p-1) /(p+1)}(1+\theta)(H+1)^{-2 /(p+1)} \leq 1 .
$$

Substituting (3.9) and (3.11) into (3.8) and using (2.1), we obtain

$$
\begin{aligned}
R(v) & \leq(1+\theta)(H+1)^{-2 /(p+1)} R(\phi ;(0,1)) \\
& =2^{-(p-1) /(p+1)}(1+\theta)(H+1)^{-2 /(p+1)} R(\phi) \\
& \leq R(\phi) .
\end{aligned}
$$

Take a $\lambda>0$ such that $\lambda v \in \mathcal{N}$. Then $\lambda v$ is not a least energy solution because $v \notin C^{1}[-1,1]$. Hence $L<R(\lambda v)=R(v)$. Therefore we conclude that

$$
L<R(v) \leq R(\phi)=L_{e} .
$$

This inequality ensures that a least energy solution cannot be even. The proof is complete.

Proof of Corollary 1.2. It is clear that the left hand side of (1.8) is less than or equal to that of (1.10). Therefore (1.10) implies (1.8). Since $H>0$, we have

$$
2^{(p-1) /(p+1)}<2\left(\frac{H+1}{2}\right)^{2 /(p+1)} .
$$

Then (1.11) implies (1.9). Therefore Theorem 1.1 proves the corollary. 


\section{Proof of EXAMPles}

In this section, we prove Examples 1.3 and 1.4.

Proof of Example [1.3. We shall verify the assumption of Corollary 1.2, Take $a=$ $1-2 \delta$. Then we have

$$
\begin{gathered}
\int_{0}^{a} h(t)(1-t)^{p+1} d t \leq \frac{\varepsilon}{p+2} \leq \frac{\delta^{p+2+c}}{p+2} \\
\int_{a}^{1} h(t)(1-t)^{p+1} d t \geq \int_{1-\delta}^{1}(1-t)^{p+1} d t=\frac{\delta^{p+2}}{p+2} .
\end{gathered}
$$

We put $\nu^{(p+1) / 2}:=2^{(p+1) / 2} \delta^{c}$. If $\delta>0$ is small enough, then $0<\nu<1$ and (1.10) holds. Since $a \rightarrow 1$ and $\nu \rightarrow 0$ as $\delta \rightarrow 0$, (1.11) clearly holds. Therefore a least energy solution is not even.

Proof of Example 1.4. First, we fix $a \in(0,1)$ such that

$$
2-2^{(p-1) /(p+1)}<a<1 .
$$

Next, we put $\alpha=\max _{|t| \leq a} g(t)$ and choose $\beta>0$ such that $\alpha<\beta<g(1)$. Lastly, we determine $b \in(a, 1)$ such that $\beta<g(t)$ in $(b, 1)$. From easy calculations, it follows that

$$
\begin{gathered}
\int_{0}^{a} h(t)(1-t)^{p+1} d t \leq \frac{\alpha^{\lambda}}{p+2} \\
\int_{a}^{1} h(t)(1-t)^{p+1} d t \geq \int_{b}^{1} h(t)(1-t)^{p+1} d t \geq \frac{\beta^{\lambda}}{p+2}(1-b)^{p+2} .
\end{gathered}
$$

We put $\nu^{(p+1) / 2}:=2^{(p+1) / 2}(1-b)^{-p-2}(\alpha / \beta)^{\lambda}$. Then $0<\nu<1$ for $\lambda>0$ large enough and (1.10) holds. Since $\nu>0$ is small enough, (4.1) implies (1.11). Consequently, Corollary 1.2 ensures the conclusion.

\section{REFERENCES}

[1] A. Ambrosetti and P. H. Rabinowitz, Dual variational methods in critical point theory and applications, J. Funct. Anal. 14, 349-381 (1973). MR0370183(51:6412)

[2] H. Brezis and L. Oswald, Remarks on sublinear elliptic equations. Nonlinear Anal. 10, 55-64 (1986). MR820658(87c:35057)

[3] J. Byeon and Z.-Q. Wang, On the Hénon equation: Asymptotic profile of ground states, I. Ann. Inst. H. Poincaré Anal. Non Linéaire 23, 803-828 (2006). MR:2271694 (2007g:35046)

[4] J. Byeon and Z.-Q. Wang, On the Hénon equation: Asymptotic profile of ground states, II. J. Differential Equations 216, 78-108 (2005). MR.2158918 (2006j:35068)

[5] P. Esposito, A. Pistoia and J. Wei, Concentrating solutions for the Hénon equation in $\mathbb{R}^{2}$. J. Anal. Math. 100, 249-280 (2006). MR2309528(2009b:35106)

[6] N. Hirano, Existence of positive solutions for the Hénon equation involving critical Sobolev terms. J. Differential Equations 247, 1311-1333 (2009). MR.2541412 (2010f:35121)

[7] R. A. Moore and Z. Nehari, Nonoscillation theorems for a class of nonlinear differential equations. Trans. Amer. Math. Soc. 93, 30-52 (1959). MR0111897(22:2755)

[8] A. Pistoia and E. Serra, Multi-peak solutions for the Hénon equation with slightly subcritical growth. Math. Z. 256, 75-97 (2007). MR2282260(2008b:35091)

[9] E. Serra, Non radial positive solutions for the Hénon equation with critical growth. Calc. Var. Partial Differential Equations 23, 301-326 (2005). MR.2142066 (2006f:35100)

[10] D. Smets, M. Willem and J. Su, Non-radial ground states for the Hénon equation, Commun. Contemp. Math. 4, 467-480 (2002). MR1918755(2003g:35086) 
[11] S. Tanaka, Uniqueness and nonuniqueness of nodal radial solutions of sublinear elliptic equations in a ball. Nonlinear Analysis 71, 5256-5267 (2009). MR 2560195 (2011d:35177)

[12] S. Tanaka, An identity for a quasilinear ODE and its applications to the uniqueness of solutions of BVPs. J. Math. Anal. Appl. 351, 206-217 (2009). MR2472934 (2009j:34050)

[13] P. H. Rabinowitz, Minimax methods in critical point theory with applications to differential equations, CBMS Regional Conf. Ser. in Math. 65, Amer. Math. Soc., Providence, RI, 1986. MR845785 (87j:58024)

Department of Mathematics, Faculty of Science and Engineering, Saga University, SAGA, 840-8502, JAPAN

E-mail address: kajikiya@ms.saga-u.ac.jp 